\title{
Sesamol attenuates oxidative stress, apoptosis and inflammation in focal cerebral ischemia/reperfusion injury
}

\author{
XIU-JUAN GAO ${ }^{1,2^{*}}$, GUAN-NAN XIE ${ }^{2 *}$, LEI LIU ${ }^{1,2}$, ZHI-JIAN FU $^{1}$, \\ ZONG-WANG ZHANG ${ }^{2}$ and LIANG-ZHU TENG ${ }^{3}$
}

\author{
${ }^{1}$ Department of Pain Management, Shandong Provincial Hospital Affiliated to Shandong University, Jinan, Shandong 250021; \\ ${ }^{2}$ Department of Anesthesiology, Liaocheng People's Hospital, Liaocheng, Shandong 252000; \\ ${ }^{3}$ Department of Neurosurgery, Shandong Provincial Hospital Affiliated to \\ Shandong University, Jinan, Shandong 250021, P.R. China
}

Received September 30, 2015; Accepted November 21, 2016

DOI: $10.3892 / \mathrm{etm} .2017 .4550$

\begin{abstract}
The aim of the present study was to evaluate the therapeutic potential of sesamol treatment on focal ischemia/reperfusion (I/R) injury in the rat brain. The results demonstrated that pretreatment with sesamol seven days prior to focal cerebral I/R injury had significant positive effects, including improvements in neurological deficits $(\mathrm{P}<0.05)$, and a reduction in malondialdehyde content and elevation of antioxidant levels (superoxide dismutase, glutathione and glutatione peroxidase; both $\mathrm{P}<0.05)$. Furthermore, levels of B cell lymphoma-2 (Bcl-2)-associated X protein and caspase-3 were significantly downregulated, whereas the level of Bcl-2 was effectively increased. Conversely, the mRNA expression of proinflammatory cytokines were significantly reduced in focal cerebral I/R injury rats upon sesamol intervention. Therefore, the beneficial effects of sesamol on cerebral I/R injury may be due to the reduction of oxidative stress, inhibition of apoptosis and inflammation. The findings of the present study suggest that sesamol supplementation may serve as potent adjuvant in the treatment of focal cerebral ischemia/reperfusion injury due to its neuroprotective effects.
\end{abstract}

\section{Introduction}

Globally, stroke is one of the leading causes of mortality and a major contributing factor to adult disabilities, and therefore there is a great interest in finding an effective clinical treatment. Previous reports have suggested that several biomechanisms

Correspondence to: Dr Liang-Zhu Teng, Department of Neurosurgery, Shandong Provincial Hospital Affiliated to Shandong University, 324 Wuweiqi Road, Jinan, Shandong 250021, P.R. China E-mail: tenglz@126.com

\section{${ }^{*}$ Contributed equally}

Key words: focal cerebral ischemia/reperfusion injury, oxidative stress, sesamol, apoptosis, inflammation, lipid peroxidation may be associated with the pathology of cerebral ischemic damage, such as ion imbalance, loss of ATP, increased release of excitatory neurochemical transmitters, increased generation of free radicals and apoptosis (1-3). In the context of stroke, these events may induce irreversible brain damage.

Oxidative stress is the first event in a sequence leading to neuronal loss following cerebral ischemia reperfusion (4). Essential cell components, including DNA, protein and lipids, are subject to excessive oxidative assault, leading to cell injury. Antioxidant defensive molecules, such as superoxide dismutase, glutathione and catalase are able to ameliorate the elevation of oxidants and therefore reduce damage to tissues (5).

Apoptosis-mediated cell death due to DNA damage is a major contributor to the tissue damage associated with cerebral ischemia (6-9). During cerebral ischemia-induced oxidative load, mitochondria release cytochrome $\mathrm{c}$, which leads to the activation of caspase-3 (10) and is a prelude to formation of the apoptosome complex, which encompasses apoptotic-protease activating factor-1, procaspase-9 and ATP (11). Apoptosomes induce the activation of procaspase- 9 , followed by procaspase-3 (12). Finally, active caspase-3 is associated with the process of DNA fragmentation that leads to cell death (11).

B cell lymphoma $(\mathrm{Bcl})-2$ family-related proteins are associated with regulation of the apoptotic process via the activation/inactivation of protein systems (13). Bcl-2 inhibits apoptotic events in various cells and is associated with reducing the overexpression of proapoptotic factors $(14,15)$ including Bcl-2-associated X protein (Bax), which serves an important role in inducing apoptosis $(16,17)$.

Cerebral inflammation is important in the progression of ischemic stroke (18-20). A number of preclinical reports have suggested that, during stroke, there is a marked elevation in the levels of pro-inflammatory mediators tumor necrosis factor (TNF)- $\alpha$, interleukin (IL)-1 $\beta$ and IL-6 $(21,22)$. Under these conditions, therapeutic agents which are able to limit the formation of reactive oxygen species (ROS) have been demonstrated to ameliorate brain damage following stroke-like events.

Sesamol (5-hydroxy-1,3-benzodioxole or 3,4-methylenedioxyphenol) is the predominant active component of sesame 
seed oil obtained from Sesamum indicum L. Sesamol has previously been demonstrated to possess potent antioxidant activity in ultraviolet and $\mathrm{Fe}^{3+} /$ ascorbate-induced lipid peroxidation in the rat brain (23). Furthermore, sesamol appears to act as a neuroprotective agent and exhibits a multitude of biological effects such as hepatoprotective, anti-inflammatory, anti-cancer and anti-aging properties (23-26). Based on this, the present study aimed to evaluate the efficacy of sesamol in alleviating cerebral ischemic injury in a rat model of middle cerebral artery occlusion (MCAO).

\section{Materials and methods}

Animals. A total of 30 male Sprague Dawley rats with a weight range of 170-200 $\mathrm{g}$ were obtained from the animal facility of Shandong University (Shandong, China). Rats were housed under standard laboratory conditions with relative humidity $55 \pm 5 \%$, temperature $25 \pm 2{ }^{\circ} \mathrm{C}$, a 12 -h light/dark cycle. Rats were fed standard diet pellets and water was provided ad libitum. Rats were randomly divided into three groups ( $n=10$ per group): Sham group; MCAO group, rats underwent MCAO procedure and treated with normal saline vehicle; and the $\mathrm{MCAO}+$ sesamol group, underwent $\mathrm{MCAO}$ procedure and treated with $25 \mathrm{mg} / \mathrm{kg} /$ day sesamol. Sesamol was administered for seven consecutive days prior to the induction of MCAO. Sham-operated and MCAO vehicle groups were administered saline under the same conditions.

MCAO model. Focal cerebral ischemic injuries were induced via an intraluminal filament surgical procedure, as previously described (27). In brief, rats were anesthetized via intraperitoneal administration of $10 \%$ chloral hydrate $(350$ mg; Sigma-Aldrich; Merck Millipore, Darmstadt, Germany). Under aseptic conditions, a small incision was made in the neck and the external carotid artery (ECA) and internal carotid artery (ICA) were exposed and isolated. A sterile nylon thread (15 $\mathrm{mm}$ long and $0.15 \mathrm{~mm}$ in diameter) was introduced from the ECA into the ICA to occlude the origin of the left middle cerebral artery. The procedure was terminated when mild resistance was felt. Nylon thread was removed $2 \mathrm{~h}$ post-surgery to restore blood supply for $24 \mathrm{~h}$ reperfusion.

Neurological evaluation. Neurological evaluation was performed at $24 \mathrm{~h}$ post-surgery. An established scoring system reported by Garcia et al (28) was used, in which six individual tests are performed and their scores are added together. The six tests conducted evaluated spontaneous activity, symmetry in limb movement, forepaw outstretching, climbing, body proprioception, and response to vibrissae stimulation. Neurological deficits were assessed by a 'blinded' assessor. Each test was scored from 0 to 3 , with a minimum neurological score of 0 and a maximum of 18. A lower score was considered to represent serious neurological deficits. All sham-operated animals had a score of 18 (28).

Evaluation of oxidative stress. Following neurological evaluation, rats were sacrificed via intraperitoneal administration of xylazine and ketamine (10 and $75 \mathrm{mg} / \mathrm{kg}$; Sigma-Aldrich; Merck Millipore). The ischemic hemispheres ( $\mathrm{n}=10$ per group) were harvested and homogenized in $4^{\circ} \mathrm{C}$ Tris buffer $(\mathrm{pH} 7.4$;
Sima-Aldrich; Merck Millipore). The homogenate was centrifuged at $2,000 \times \mathrm{g}$ at $4^{\circ} \mathrm{C}$ for $15 \mathrm{~min}$ and the resulting supernatant was evaluated to determine the content of malondialdehyde (MDA), activity of superoxide dismutase (SOD) and glutathione peroxidase (GPx), and levels of reduced glutathione (GSH) using a spectrophotometer (UV-2600; Shimadzu Corporation, Kyoto, Japan) and assay kits according to the manufacturer's protocol (SOD, cat. no. A001-1; MDA, A003-1; GPx, A005; GSH, A006; Nanjing Jiancheng Bioengineering Institute, Nanjing, China).

Measurement of apoptotic protein expression using western blot analysis. Ischemic hemispheres ( $\mathrm{n}=5$ per group) were harvested, weighed and homogenized in radioimmunoprecipitation assay buffer (Abcam, Cambridge, UK) containing 0.22\% $\beta$-glycerophosphate, $10 \%$ tergitol-NP40,0.18\% sodium orthovanadate, $5 \%$ sodium deoxycholate, $0.38 \%$ EGTA, $1 \%$ SDS, $6.1 \%$ Tris, $0.29 \%$ EDTA, $8.8 \%$ sodium chloride, $1.12 \%$ sodium pyrophosphate decahydrate $(\mathrm{pH} 7.5)$, at $4^{\circ} \mathrm{C}$. The lysate was collected and placed in an orbital shaker for $2 \mathrm{~h}$ at $4^{\circ} \mathrm{C}$. Solubilised proteins were collected from the supernatant following centrifugation at $16,000 \mathrm{x}$ g for $20 \mathrm{~min}$ at $4^{\circ} \mathrm{C}$, and $20 \mu \mathrm{g}$ samples were subjected to $10 \%$ SDS-PAGE (Sigma-Aldrich; Merck Millipore) and electrotransferred to nitrocelluose membranes (pore size, $0.45 \mathrm{~mm}$; Sigma-Aldrich; Merck Millipore) to measure Caspase-3, Bax and Bcl-2. Membranes were blocked using 5\% nonfat dry milk dissolved in $0.05 \mathrm{~mol} / 1 \mathrm{TBS}$ containing $200 \mathrm{mmol} / 1$ (pH 7.4), and washed three times in TBS (30 min each). The primary antibodies used in the study were as follows; Caspase-3 $(1: 1,000$; cat. no. 19677-1-AP), Bax, (1:1,000; cat. no. 50599-2-Ig) and Bcl-2 (1:750; cat. no. 12789-1-AP; all ProteinTech Group, Inc., Chicago, IL, USA). The blot was washed with TBS and incubated for $1 \mathrm{~h}$ at room temperature with goat anti-mouse antibody conjugated to peroxidase (1:1,500; cat. no. sc-2005; Santa Cruz Biotechnology, Inc., Dallas, TX, USA). $\beta$-actin (1:5,000; Santa Cruz Biotechnology, Inc.) was used as a control. Binding of the antibodies was detected using an ECL detection kit (Applygen Technologies, Inc., Beijing, China) and protein bands were visualized using the Gel Doc XR system (Bio-Rad Laboratories, Inc., Hercules, CA, USA) and quantified using Image J software (version 1.50; National Institutes of Health, Bethesda, MD, USA). All blots were performed in triplicate.

Analysis of inflammatory cytokine gene expression by reverse transcription-quantitative polymerase chain reaction. RNA was isolated from the frozen rat ischemic hemispheres and purified using RNase (Sigma-Aldrich; Merck Millipore) according to the manufacturer's protocol. Genomic DNA was removed by the addition of $10 \mu 110 \mathrm{X}$ DNase buffer and $1 \mu \mathrm{l}$ DNase I (both Sigma-Aldrich; Merck Millipore) to the RNA sample $(2 \mu \mathrm{l})$, which was subsequently incubated for $30 \mathrm{~min}$ at $37^{\circ} \mathrm{C}$. cDNA was synthesized from $1 \mu \mathrm{g}$ of the purified RNA sample using oligo-dT primers, dNTPs, DTT, RNase inhibitor and reverse transcriptase (all Santa Cruz Biotechnology, Inc.) according to the manufacturer's protocol. The oligonucleotide primers used are displayed in Table I. A total of $1 \mu \mathrm{l}$ forward primer and $1 \mu 1$ reverse primer were added to $10 \mu \mathrm{l}$ MasterMix (Santa Cruz Biotechnology, Inc.), and diluted with water to a total volume of $15 \mu \mathrm{l}$. An additional $0.5 \mathrm{ml}$ MasterMix was added followed by $1 \mu 1$ 
cDNA, and the mixture was vortexed prior to PCR. The PCR reactions were performed using a LightCycler 480 Real-Time PCR System (Roche Diagnostics, Basel, Switzerland) and consisted of an initial denaturation at $95^{\circ} \mathrm{C}$ for $3 \mathrm{~min}$, followed by 35 cycles at $95^{\circ} \mathrm{C}$ for $50 \mathrm{sec}, 58^{\circ} \mathrm{C}$ for $45 \mathrm{sec}$ and $72^{\circ} \mathrm{C}$ for $50 \mathrm{sec}$. The reactions were terminated via an elongation step at $72^{\circ} \mathrm{C}$ for $7 \mathrm{~min}$. The integrated densities value was analyzed using a computerized image analysis system (Motic Images Advanced 3.2; Motic Instruments, Inc., Richmond, $\mathrm{BC}$, Canada). Data was quantified via the $2^{-\Delta \Delta \mathrm{Cq}}$ method (29). All tests were performed in triplicate.

Statistical analysis. Data are presented as the mean \pm standard error of the mean. Statistical analysis of the data was performed using SPSS, version 13.0 (SPSS, Inc., Chicago, IL, USA). All data was analysed by one-way analysis of variance followed by the Tukey test for multiple comparisons. $\mathrm{P}<0.05$ was considered to indicate a statistically significant difference.

\section{Results}

Effect of sesamol and MCAO on neurological score. Neurological scores were significantly decreased in the MCAO group compared with the sham group $(\mathrm{P}<0.05)$. Following sesamol treatment, neurological deficits were significantly reduced compared with those in the MCAO group $(\mathrm{P}<0.05$; Fig. 1).

Effect of sesamol and MCAO on lipid peroxidation. Lipid peroxidation in the ischemic hemisphere was quantified by evaluating MDA levels (Fig. 2). The brain level of MDA in rats from the MCAO group was significantly higher than in the sham rat group $(\mathrm{P}<0.05)$, indicating elevated in vivo oxidative stress in the brain. This increase was significantly decreased by treatment with sesamol compared with MCAO rats $(\mathrm{P}<0.05)$.

Effect of sesamol and MCAO on antioxidant level. In the present study, there was a significant decline in the level of antioxidants (GSH, SOD and GPx) following the induction of MCAO compared with the sham rats $(\mathrm{P}<0.05)$. Administration of sesamol significantly $(\mathrm{P}<0.05)$ increased the level of antioxidants in the brain through its anti-lipid peroxidative effect (Fig. 3).

Influence of sesamol on the expression of apoptosis-related proteins following MCAO injury. Protein levels of Caspase-3, $\mathrm{Bax}$ and $\mathrm{Bcl}-2$ in ischemic cortex tissues were assesed using western blot analysis following $24 \mathrm{~h}$ of reperfusion (Fig. 4A). A significant upregulation in protein expression of Caspase-3 and $\mathrm{Bax}$, and a significant downregulation in $\mathrm{Bcl}-2$ protein expression were observed in the MCAO group (all $\mathrm{P}<0.05$ ). However, rats treated with sesamol exhibited a significant downregulation in Caspase- 3 and Bax expression and a significant upregulation in $\mathrm{Bcl}-2$ protein expression $(\mathrm{P}<0.05$; Fig. 4B-D).

Effect of MCAO and sesamol on cytokine mRNA expression profile. In the present study, MCAO groups exhibited a significant upregulation in mRNA expression of the proinflammatory cytokines TNF- $\alpha$ and IL-6 $(\mathrm{P}<0.05)$, whereas inflammation
Table I. List of primers used for reverse transcription-quantitative polymerase chain reaction.

\begin{tabular}{lc}
\hline Gene & \multicolumn{1}{c}{ Primer sequence } \\
\hline IL-6 & F: 5'-ATCTGCCCTTCAGGAACAGC-3' \\
& R: 5'-AGCCTCCGACTTGTGAAGTG 3' \\
TNF- $\alpha$ & F: 5'-CTTCTC CTT CCT GAT CGT GG-3' \\
& R: 5'-GCTGGTTATCTCTCAGCTCCA-3' \\
$\beta$-actin & F: 5'-GTGGGGCGCCCAGGCACCA-3' \\
& R: 5'-GCTCGGCCGTGGTGGTGAAGC-3'
\end{tabular}

IL-6, interleukin-6; TNF- $\alpha$, tumour necrosis factor- $\alpha$; F, forward; $\mathrm{R}$, reverse.

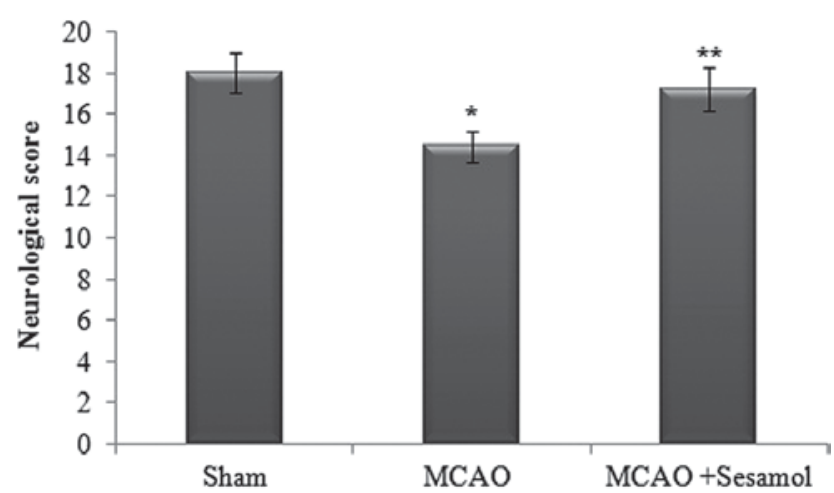

Figure 1. Effect of MCAO and sesamol on neurological score at $24 \mathrm{~h}$ post-surgery. The neurological assessment was performed using the 18-point Garcia scale system. Values are presented as the mean \pm standard error of the mean for each group. ${ }^{*} \mathrm{P}<0.05$ vs. sham group, ${ }^{* *} \mathrm{P}<0.05$ vs. MCAO group. $\mathrm{MCAO}$, middle cerebral artery occlusion.

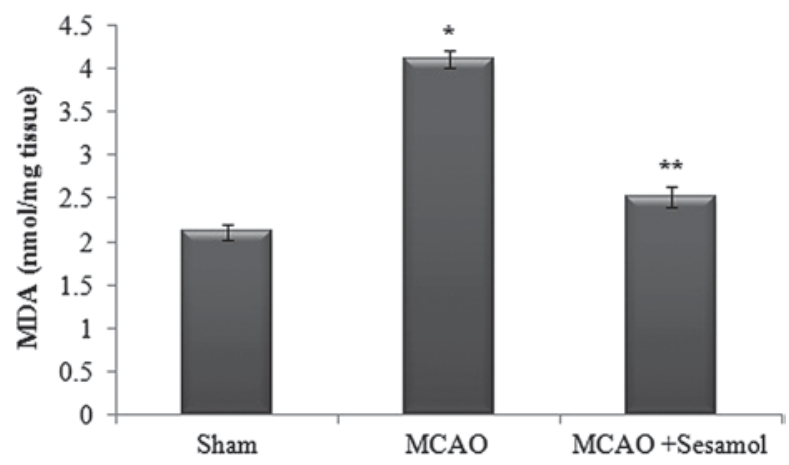

Figure 2. Effect of MCAO and sesamol on oxidative stress marker MDA in ischemic cortex tissue. Values are presented as the mean \pm standard error of the mean for 10 rats in each group. ${ }^{*} \mathrm{P}<0.05$ vs. sham group, ${ }^{* *} \mathrm{P}<0.05$ vs. MCAO group. MCAO, middle cerebral artery occlusion; MDA, malondialdehyde.

was mitigated in rats treated with sesamol due to a significant downregulation of the mRNA expression of TNF- $\alpha$ and IL-6 $(\mathrm{P}<0.05$; Fig. 5A). Furthermore, the relative expression levels of TNF- $\alpha$ and IL-6 were significantly higher in the MCAO group compared with the control group $(\mathrm{P}<0.05)$, and sesamol treatment induced a significant reduction in the expression of proinflammatory cytokines $(\mathrm{P}<0.05$; Fig. $5 \mathrm{~B})$. 

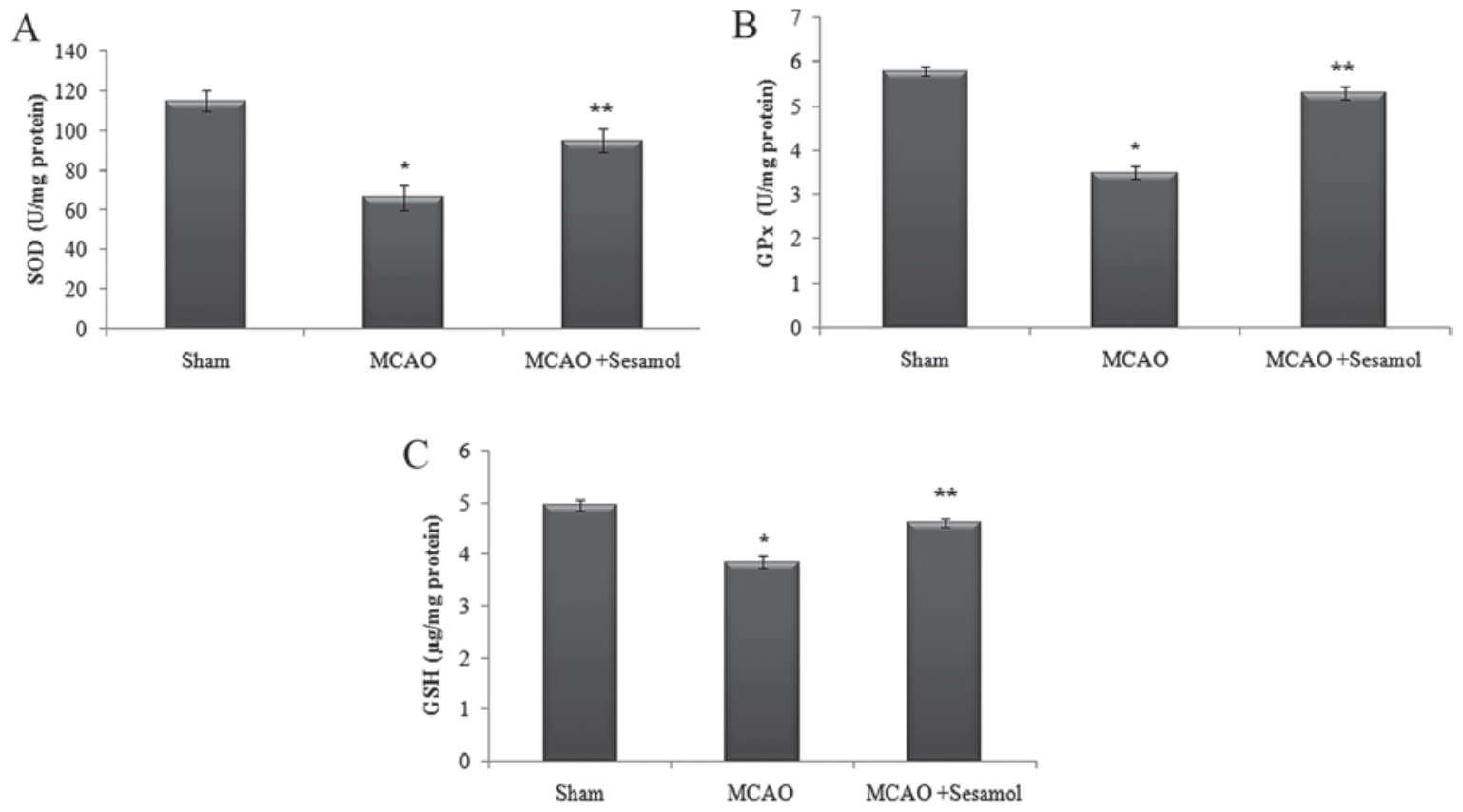

Figure 3. Effect of MCAO and sesamol on antioxidant levels in ischemic cortex tissue. (A) SOD, (B) GPx and (C) GSH. Values are presented as the mean \pm standard error of the mean for 10 rats in each group. ${ }^{*} \mathrm{P}<0.05$ vs. sham group, ${ }^{* *} \mathrm{P}<0.05$ vs. MCAO group. MCAO, middle cerebral artery occlusion; SOD, superoxide dismutase; GPx, glutathione peroxidase; GSH, reduced glutathione.
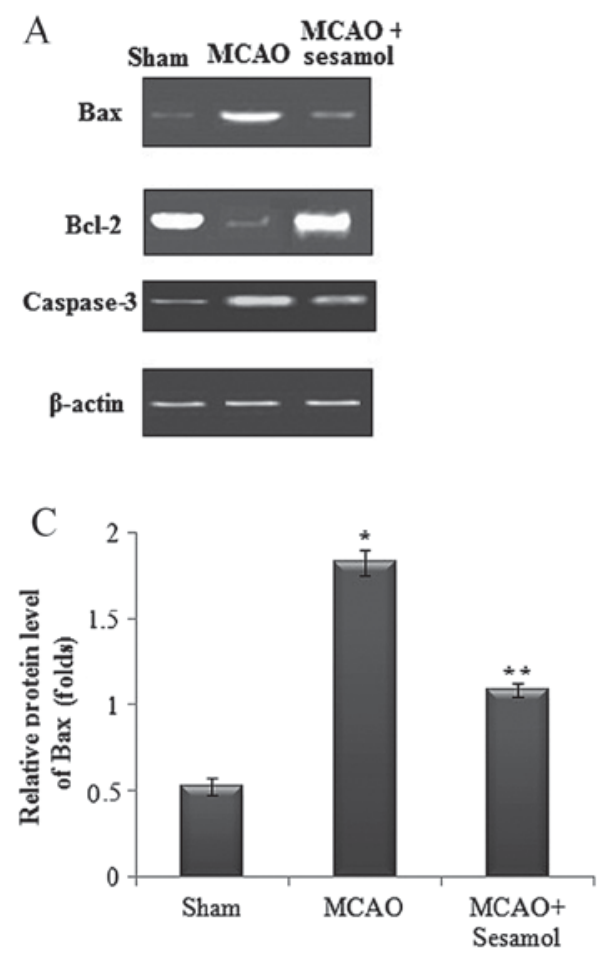

B

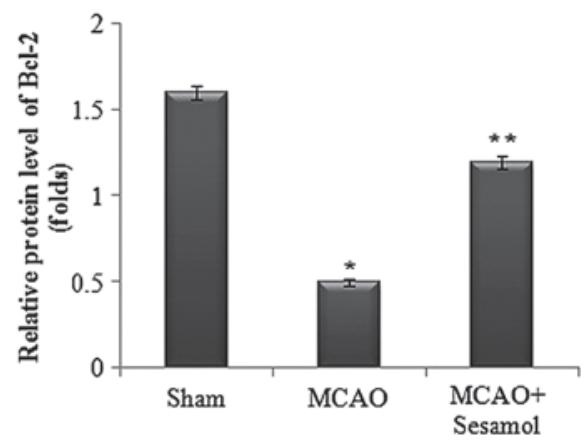

$\mathrm{D}$

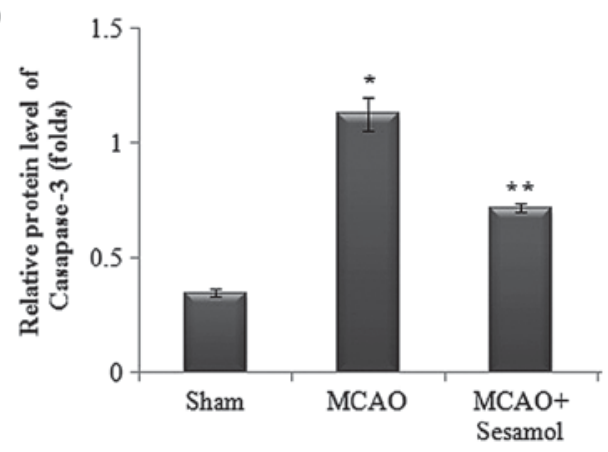

Figure 4. Effect of MCAO and sesamol on protein expression of apoptotic markers, Bax, Bcl-2 and caspase-3 in the ischemic cortex tissue. (A) MCAO rats exhibited increased Bax and caspase-3 expression, whereas Bcl-2 expression was decreased. (B-D) Sesamol significantly reduced apoptosis and restored marker levels to normalcy. Values are presented as the mean \pm standard error of the mean for five rats in each group. ${ }^{*} \mathrm{P}<0.05$ vs. sham group, ${ }^{* *} \mathrm{P}<0.05$ vs. MCAO group. MCAO, middle cerebral artery occlusion; Bcl-2, B cell lymphoma 2; Bax, Bcl-2-associated X protein.

\section{Discussion}

Stroke is a leading cause of morbidity and mortality worldwide $(30,31)$. The reduction of blood flow to the brain that occurs during cerebral ischemia may aggravate the necrosis of neuronal and brain cells $(32,33)$. It has been demonstrated that various pathological mechanisms, such as elevation of ROS levels, inhibition of the detoxification cascade and the loss of antioxidant reserve enzymes are associated with the progression of cerebral ischemia $(34,35)$. Preclinical MCAO models exhibit an identical pattern of ischemic brain damage as is observed in human patients who have suffered an ischemic stroke (36). The 

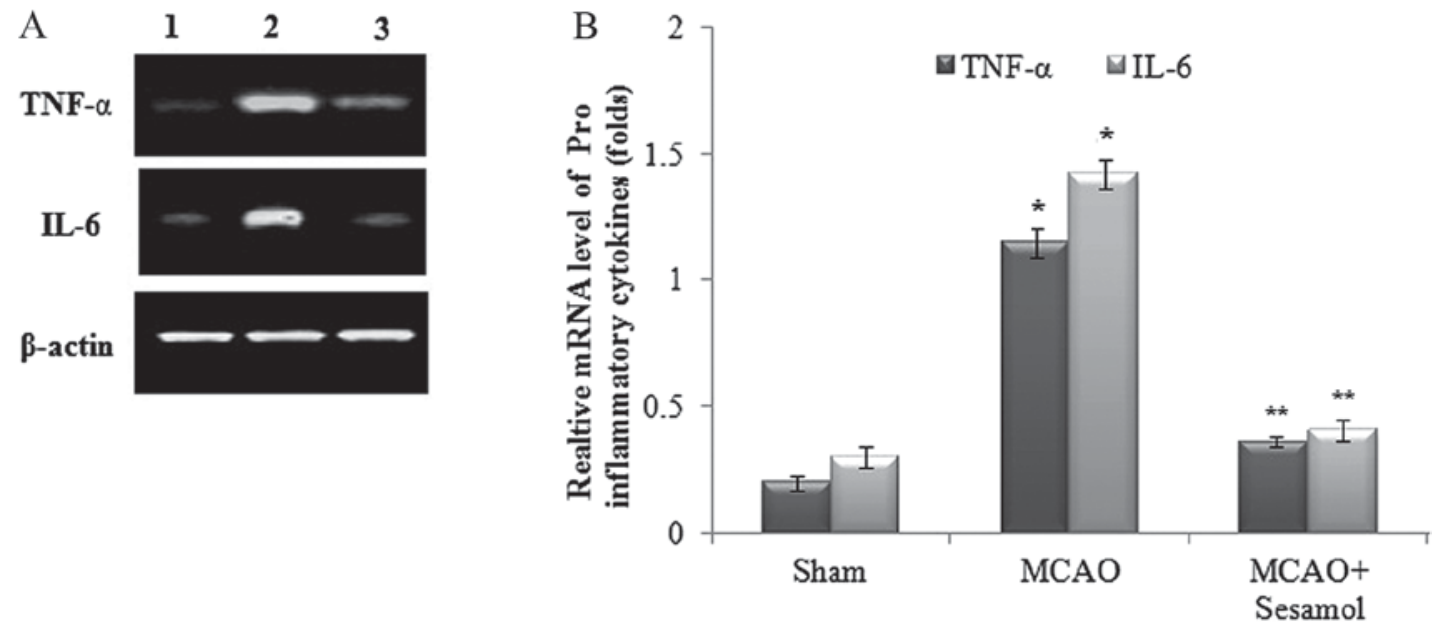

Figure 5. Reverse transcription-quantitative polymerase chain reaction analysis of pro-inflammatory cytokines in ischemic cortex tissues with $\beta$-actin as an internal control. (A) Following MCAO, mRNA expression of TNF- $\alpha$ and IL- 6 was upregulated and this effect was reduced by sesamol treatment. Lane 1, Sham; Lane 2, MCAO; Lane 3, MCAO + sesamol. (B) Relative mRNA expression of TNF- $\alpha$ and IL-6. Values are presented as the mean \pm standard error of the mean for five rats in each group. ${ }^{*} \mathrm{P}<0.05$ vs. sham group, ${ }^{* *} \mathrm{P}<0.05$ vs. MCAO group. MCAO, middle cerebral artery occlusion; TNF- $\alpha$, tumour necrosis factor- $\alpha$; IL-6, interleukin-6.

aim of the present study was to evaluate the protective impact of sesamol on focal cerebral ischemic reperfusion injury.

In the present study, MCAO rats exhibited a significant reduction in the voluntary motor activity and the flexion tests. This may be associated with excessive free radical generation in the hippocampus and cortical areas, which are responsible for motor activities (37). Sesamol administration was able to significantly reduce the neurological deficts and restore the brain motor activities, which supports the findings of a previous report (38).

The ROS generated during cerebral ischemia are able to oxidize biomolecules such as lipids, which further increases cell toxicity and brain dysfunction. The detrimental effects of free radicals on lipids lead to the formation of the final lipid peroxidation product MDA, which is a toxic adduct that induces neuronal cell death and loss of brain activity (39). In the present study, sesamol treatment was able to reduce MDA levels in MCAO rats, which may be due to the inhibitory effect of sesamol on oxidative damage (40). GSH, a vital intracellular non-protein thiol, acts as a scavenger of free radicals (41). Previous studies have demonstrated that cerebral ischemia mediated lipid peroxidation is concurrent with GSH depletion in brain tissue (42-44). Furthermore, it has previously been suggested that antioxidant enzymes such as SOD and GPx may serve a key role in the regulation of redox homeostasis in tissues $(45,46)$. In the present study, MCAO groups displayed significant reductions in, SOD and GPx levels in the brain ischemic tissue, compared with a sham group. Treatment with sesamol was able to restore depleted antioxidant levels due to its potent antioxidant activity and anti-lipid peroxidative effect. The effect of sesamol may be attributed to the presence of free phenolic hydroxyl groups (47).

The levels of proinflammatory cytokines such as TNF- $\alpha$ and IL-1 $\beta$ are markedly higher during ischemic stroke, which also contributes to brain damage associated with stroke $(48,49)$. Furthermore, elevated levels of proinflammatory cytokines are able to augment the expression of intracellular adhesion molecule-1 and E-selectin in leukocytes, and enhance the adhesion and transendothelial migration of leukocytes (19). These mechanisms induce an inflammatory cascade, which leads to a loss of blood-brain barrier integrity and induces fluid accumulation in the brain, resulting in neuronal cell death (50). In the present study, MCAO rats exhibited an upregulation in TNF- $\alpha$ and IL- $1 \beta$ mRNA expression in ischemic brain tissue. These results are corroborated by previous studies, which reported that exacerbated TNF- $\alpha$ and IL-1 $\beta$ levels may be important factors in reperfusion injury following transient brain ischemia (51); however, sesamol treatment significantly downregulated the expression of proinflammatory cytokines via its anti-inflammatory activity $(52,53)$.

Apoptosis serves an important role in the etiology of cerebral ischemia reperfusion injury via activating the apoptotis-related protein cascade located within apoptotic cells $(54,55)$. Bcl-2 family-related proteins are also associated with the regulation of major apoptotic signal transduction pathways and are able to cause irreversible cell damage $(56,57)$, and the $\mathrm{Bcl}-2$ /Bax ratio is important in the regulation of apoptotic cell death (58). Furthermore, Caspase- 3 is the primary protein associated with apoptosis and is vital for inducing DNA fragmentation and apoptosis-related cell morphology changes $(59,60)$. In the present study, MCAO rats exhibited an upregulation in Caspase-3 and Bax expression, and a downregulation in $\mathrm{Bcl}-2$ expression in ischemic tissue. Sesamol treatment was able to restore the expression of apoptotic markers to normal levels and therefore prevent apoptosis from occurring. The anti-apoptotic activity of sesamol has been suggested by previous results $(61,62)$.

In conclusion, the results of the present study suggest that the neuroprotective effect of sesamol on cerebral tissue exposed to MCAO-reperfusion and ischemic damage in rats is mainly due to the inhibition of neurological deficits and lipid peroxidation, the restoration of antioxidants and the mitigation of inflammation and apoptosis responses. Based on the results of the present study, sesamol may be a suitable candidate for 
the clinical treatment of various brain ischemic conditions resulting from oxidative stress. Furthermore, future research into the molecular mechanisms of sesamol may reveal the extent of its anti-stroke effect.

\section{References}

1. Dirnagl U, Iadecola C and Moskowitz MA: Pathobiology of ischaemic stroke: An integrated view. Trends Neurosci 22: 391-397, 1999.

2. Durukan A and Tatlisumak T: Acute ischemic stroke: Overview of major experimental rodent models, pathophysiology, and therapy of focal cerebral ischemia. Pharmacol Biochem Behav 87: 179-197, 2007.

3. Lapchak PA and Araujo DM: Advances in ischemic stroke treatment: Neuroprotective and combination therapies. Exp Opin Emer Drugs 12: 97-112, 2007.

4. Chan PH: Reactive oxygen radicals in signaling and damage in the ischemic brain. J Cereb Blood Flow Metab 21: 2-14, 2001.

5. Chen H, Yoshioka H, Kim GS, Jung JE, Okami N, Sakata H, Maier CM, Narasimhan P, Goeders CE and Chan PH: Oxidative stress in ischemic brain damage: Mechanisms of cell death and potential molecular targets for neuroprotection. Antioxid Redox Signal 14: 1505-1517, 2011.

6. Kawaguchi M, Drummond JC, Cole DJ, Kelly PJ, Spurlock MP and Patel PM: Effect of isoflurane on neuronal apoptosis in rats subjected to focal cerebral ischemia. Anesth Analg 98: 798-805, 2004.

7. Liu R, Gao M, Yang ZH and Du GH: Pinocembrin protects rat brain against oxidation and apoptosis induced by ischemia-reperfusion both in vivo and in vitro. Brain Res 1216: 104-115, 2008

8. Wang W, Xu J, Li L, Wang P, Ji X, Ai H, Zhang L and Li L: Neuroprotective effect of morroniside on focal cerebral ischemia in rats. Brain Res Bull 83: 196-201, 2010.

9. Lu Y, Zhang J, Ma B, Li K, Li X, Bai H, Yang Q, Zhu X, Ben J and Chen Q: Glycine attenuates cerebral ischemia/reperfusion injury by inhibiting neuronal apoptosis in mice. Neurochem Int 61: 649-658, 2012

10. Broughton BR, Reutens DC and Sobey CG: Apoptotic mechanisms after cerebral ischemia. Stroke 40: e331-e339, 2009.

11. Wang X: The expanding role of mitochondria in apoptosis. Genes Dev 15: 2922-2933, 2001

12. Li P, Nijhawan D, Budihardio I, Srinivasula SM, Ahmad M, Alnemri ES and Wang X: Cytochrome $\mathrm{c}$ and dATP dependent formation of Apaf-1/caspase-9 complex initiates an apoptotic protease cascade. Cell 91: 479-489, 1997.

13. Elmore S: Apoptosis: A review of programmed cell death. Toxicol Pathol 35: 495-516, 2007.

14. Gross A, McDonnell JM and Korsmeyer SJ: BCL-2 family members and the mitochondria in apoptosis. Genes Dev 13 1899-1911, 1999.

15. Shigeomi S, Narita M and Yoshihide T: Bcl-2 family proteins regulate the release of apoptogenic cytochrome $\mathrm{c}$ by the mitochondrial channel VDAC. Nature 399: 483-487, 1999.

16. Hsu YT, Wolter KG and Youle RJ: Cytosol-to-membrane redistribution of Bax and Bcl-X(L) during apoptosis. Proc Nat Acad Sci USA 94: 3668-3672, 1997.

17. Martinou JC and Youle RJ: Mitochondria in apoptosis: Bcl-2 family members and mitochondrial dynamics. Dev Cell 21 : 92-101, 2011.

18. Amantea D, Nappi G, Bernardi G, Bagetta G and Corasaniti MT: Post-ischemic brain damage: Pathophysiology and role of inflammatory mediators. FEBS J 276: 13-26, 2009.

19. Jin R, Yang G and Li G: Inflammatory mechanisms in ischemic stroke: Role of inflammatory cells. J Leukoc Biol 87: 779-789, 2010.

20. Kleinig TJ and Vink R: Suppression of inflammation in ischemic and hemorrhagic stroke: Therapeutic options. Curr Opin Neurol 22: 294-301, 2009.

21. Wang Q, Tang XN and Yenari MA: The inflammatory response in stroke. J Neuroimmunol 184: 53-68, 2007.

22. del Zoppo G, Ginis I, Hallenbeck JM, Iadecola C, Wang X and Feuerstein GZ: Inflammation and stroke: Putative role for cytokines, adhesion molecules and iNOS in brain response to ischemia. Brain Pathol 10: 95-112, 2000.
23. Prasad NR, Mahesh T, Menon VP, Jeevanram RK and Pugalendi KV: Photo protective effect of sesamol on UVB-radiation induced oxidative stress inhuman blood lymphocytes in vitro. Environ Toxicol Pharmacol 20: 1-5, 2005.

24. Hou RC, Chen YS, Chen CH, Chen YH and Jeng KC: Protective effect of 1,2,4-benzenetriol on LPS-induced NO production by BV2 microglial cells. J Biomed Sci 13: 89-99, 2006.

25. Hsu DZ, Chen KT, Li YH, Chuang YC and Liu MY: Sesamol delays mortality and attenuates hepatic injury after cecal ligation and puncture in rats: Role of oxidative stress. Shock 25: 528-532, 2006.

26. Sharma S and Kaur IP: Development and evaluation of sesamol as an antiaging agent. Int J Dermatol 45: 200-208, 2006.

27. Longa EZ, Weinstein PR, Carlson S and Cummins R: Reversible middle cerebral artery occlusion without craniectomy in rats. Stroke 20: 84-91, 1989.

28. Garcia J, Wagner S, Liu KF and Hu XJ: Neurological deficit and extent of neuronal necrosis attributable to middle cerebral artery occlusion in rats. Statistical validation. Stroke 26: 627-635, 1995.

29. Livak KJ and Schmittgen TD: Analysis of relative gene expression data using real-time quantitative PCR and the 2(-Delta Delta C (T)) Method. Methods 25: 402-408, 2001.

30. Bora KS and Sharma A: Neuroprotective effect of Artemisia absinthium L. on focal ischemia and reperfusion-induced cerebral injury. J Ethnopharmacol 129: 403-409, 2010.

31. Hankey GJ: The global and regional burden of stroke. Lancet Global Health 1: e239-e240, 2013.

32. Traystman RJ: Animal models of focal and global cerebral ischemia. ILARJ 44: 85-95, 2003.

33. Park SJ, Nam KW, Lee HJ, Cho EY, Koo U and Mar W: Neuroprotective effects of an alkaloid-free ethyl acetate extract from the root of Sophora flavescens Ait. against focal cerebral ischemia in rats. Phytomedicine 16: 1042-1051, 2009.

34. Kutsuna S, Tsuruta R, Fujita M, Todani M, Yagi T, Ogino Y, Igarashi M, Takahashi K, Izumi T, Kasaoka S, et al: Cholinergic agonist physostigmine suppresses excessive superoxide anion radical generation in blood, oxidative stress, early inflammation, and endothelial injury in rats with forebrain ischemia/reperfusion. Brain Res 1313: 242-249, 2010.

35. Pratap R, Pillai KK, Khanam R, Islam F, Ahmad SJ and Akhtar M: Protective effect of irbesartan, an angiotensin II receptor antagonist, alone and in combination with aspirin on middle cerebral artery occlusion model of focal cerebral ischemia in rats. Hum Exp Toxicol 30: 354-362, 2011.

36. Ginsberg MD and Busto R: Rodent models of cerebral ischemia. Stroke 20: 1627-1642, 1989.

37. Kumar P, Kalonia H and Kumar A: Sesamol attenuate 3-nitropropionic acid-induced Huntington-like behavioral, biochemical, and cellular alterations in rats. J Asian Nat Prod Res 11: 439-450, 2009.

38. Cohen GM: Caspases: The executioners of apoptosis. Biochem J 326: 1-16, 1997.

39. Serteser M, Ozben T, Gumuslu S, Balkan S and Balkan E: Lipid peroxidation in rat brain during focal cerebral ischemia: Prevention of malondialdehyde and lipid conjugated diene production by a novel antiepileptic, lamotrigine. Neurotoxicology 23: 111-119, 2002.

40. Misra S, Tiwari V, Kuhad A and Chopra K: Modulation of nitrergic pathway by sesamol prevents cognitive deficits and associated biochemical alterations in intracerebroventricular streptozotocin administered rats. Eur J Pharmacol 659: 177-186, 2011.

41. Liu Z, Li P, Zhao D, Tang H and Guo J: Protective effect of extract of Cordyceps sinensis in middle cerebral artery occlusion-induced focal cerebral ischemia in rats. Behav Brain Funct 6: 61, 2010.

42. Chan PH: Reactive oxygen radicals in signaling and damage in the ischemic brain. J Cereb Blood Metab 21: 2-14, 2001

43. Al-Omar FA, Nagi MN, Abdulgadir MM, Al Joni KS and Al-Majed AA: Immediate and delayed treatments with curcumin prevent forebrain ischemia-induced neuronal damage and oxidative insult in the rat hippocampus. Neurochem Res 31: 611-618, 2006.

44. Saleem S, Ahmad M, Ahmad AS, Yousuf S, Ansari MA, Khan MB, Ishrat T and Islam F: Behavioral and histologic neuroprotection of aqueous garlic extract after reversible focal cerebral ischemia. J Med Food 9: 537-544, 2006.

45. Gilgun-Sherki Y, Rosenbaum Z, Melamed E and Offen D: Antioxidant therapy in acute central nervous system injury: Current state. Pharmacol Rev 54: 271-284, 2002. 
46. Lo EH, Dalkara T and Moskowitz MA: Mechanisms, challenges and opportunities instroke. Nat Rev Neurosci 4: 399-415, 2003.

47. Uchida M, Nakajin S, Toyoshima S and Shinoda M: Antioxidative effect of sesamol and related compounds on lipid peroxidation. Biol Pharm Bull 19: 623-626, 1996.

48. Hallenbeck JM: The many faces of tumor necrosis factor in stroke. Nat Med 8: 1363-1368, 2002.

49. Rothwell N, Allan S and Toulmond S: The role of interleukin 1 in acute neu-rodegeneration and stroke: Pathophysiological and therapeutic implications. J Clin Invest 100: 2648-2652, 1997.

50. Huang J, Upadhyay UM and Tamargo RJ: Inflammation in stroke and focal cerebral ischemia. Surg Neurol 66: 232-245, 2006.

51. Tang NY, Liu CH, Hsieh CT and Hsieh CL: The anti-inflammatory effect of paeoniflorin on cerebral infarction induced by ischemia-reperfusion injury in Sprague-Dawley rats. Am J Chin Med 38: 51-64, 2010.

52. Hsu DZ, Chen YW, Chu PY, Periasamy S and Liu MY: Protective effect of 3,4-methylenedioxyphenol (Sesamol) on stress-related mucosal disease in rats. Biomed Res Int 2013: 481827, 2013.

53. Hassanzadeh P, Arbabi E and Rostami F: The ameliorative effects of sesamol against seizures, cognitive impairment and oxidative stress in the experimental model of epilepsy. Iran J Basic Med Sci 17: 100-107, 2014

54. Galluzzi L, Morselli E, Kepp O and Kroemer G: Targeting post-mitochondrial effectors of apoptosis for neuroprotection. Biochim Biophys Acta 1787: 402-413, 2009.

55. Nakka VP, Gusain A, Mehta SL and Raghubir R: Molecular mechanisms of apoptosis in cerebral ischemia: Multiple neuroprotective opportunities. Mol Neurobiol 37: 7-382, 2008.
56. Weyhenmeyer B, Murphy AC, Prehn JH and Murphy BM: Targeting the anti-apoptotic Bcl-2 family members for the treatment of cancer. Exp Oncol 34: 192-199, 2012.

57. Yang TM, Barbone D, Fennell DA and Broaddus VC: Bcl-2 family proteins contribute to apoptotic resistance in lung cancer multicellular spheroids. Am J Respir Cell Mol Biol 41: 14-23, 2009.

58. Bar-Am O, Weinreb O, Amit T and Youdim MB: Regulation of Bcl-2 family proteins, neurotrophic factors, and APP processing in the neuro rescue activity of propargylamine. FASEB J 19: 1899-1901, 2005.

59. Jänicke RU, Sprengart ML, Wati MR and Porter AG: Caspase-3 is required for DNA fragmentation and morphological changes associated with apoptosis. J Biol Chem 273: 9357-9360, 1998.

60. Ramachandran S and Prasad NR: Sesamol modulates ultraviolet-B-induced apoptotic and inflammatory signalling in human skin dermal fibroblasts. Int J Nutr Pharmacol Neurol Dis 2: 31-39, 2012.

61. Raza SS, Khan MM, Ahmad A, Ashafaq M, Khuwaja G, Tabassum R, Javed H, Siddiqui MS, Safhi MM and Islam F: Hesperidin ameliorates functional and histological outcome and reduces neuroinflammation in experimental stroke. Brain Res 1420: 93-105, 2011.

62. Nayak PG, Paul P, Bansal P, Kutty NG and Pai KS: Sesamol prevents doxorubicin-induced oxidative damage and toxicity on H9c2 cardiomyoblasts. J Pharm Pharmacol 65: 1083-1093, 2013. 